\title{
Single versus dual blockade of the renin-angiotensin system in patients with IgA nephropathy
}

\author{
David Paul Lennartz ${ }^{1} \cdot$ Claudia Seikrit $^{1} \cdot$ Stephanie Wied $^{2} \cdot$ Christina Fitzner $^{2} \cdot$ Frank Eitner $^{1,3} \cdot$ Ralf-Dieter Hilgers $^{2}$. \\ Thomas Rauen $^{1} \cdot$ Jürgen Floege ${ }^{1}$
}

Received: 6 May 2020 / Accepted: 11 August 2020 / Published online: 27 August 2020

(c) The Author(s) 2020

\begin{abstract}
Background Inhibitors of the renin-angiotensin system (RAS) are cornerstones of supportive therapy in patients with IgA nephropathy (IgAN). We analyzed the effects of single versus dual RAS blockaQueryde during our randomized STOP-IgAN trial.

Methods STOP-IgAN participants with available successive information on their RAS treatment regimen and renal outcomes during the randomized 3-year trial phase were stratified post hoc into two groups, i.e. patients under continuous single or dual RAS blocker therapy over the entire 3 years of the trial phase. Primary and secondary STOP-IgAN trial endpoints, i.e. frequencies of full clinical remission, eGFR-loss $\geq 15$ and $\geq 30 \mathrm{ml} / \mathrm{min} / 1.73 \mathrm{~m}^{2}$ and ESRD onset, were analyzed by logistic regression and linear mixed effects models.

Results Among the 112 patients included in the present analysis, $82(73 \%)$ were maintained on single and $30(27 \%)$ on dual RAS inhibitor therapy throughout the trial. Neither RAS blocker strategy significantly affected full clinical remission, eGFR-loss rates, onset of ESRD. Proteinuria moderately increased in patients under dual RAS blockade by $0.1 \mathrm{~g} / \mathrm{g}$ creatinine during the 3 -year trial phase. This was particularly evident in patients without additional immunosuppression during the randomized trial phase, where proteinuria increased by $0.2 \mathrm{~g} / \mathrm{g}$ creatinine in the dual RAS blockade group. In contrast, proteinuria decreased in patients under single RAS blocker therapy by $0.3 \mathrm{~g} / \mathrm{g}$ creatinine. The course of eGFR remained stable and did not differ between the RAS treatment strategies.

Conclusion In the STOP-IgAN cohort, neither RAS blocker regimen altered renal outcomes. Patients on dual RAS blockade even exhibited higher proteinuria over the 3 -year trial phase.
\end{abstract}

Keywords Renin-angiotensin system $\cdot$ RAS system $\cdot$ RAS blockers $\cdot$ IgA nephropathy $\cdot$ STOP-IgAN

\section{INTRODUCTION}

$\operatorname{Ig}$ A nephropathy (IgAN) is the most common type of glomerulonephritis in the western world [1]. It usually runs a chronic, often slowly progressive course and there is wide

David Paul Lennartz and Claudia Seikrit contributed equally to this manuscript.

Jürgen Floege

jfloege@ukaachen.de

1 Division of Nephrology and Clinical Immunology, RWTH Aachen University, Pauwelsstr. 30, 52057 Aachen, Germany

2 Department of Medical Statistics, RWTH Aachen University, Aachen, Germany

3 Kidney Diseases Research, Bayer AG, Wuppertal, Germany consensus that blood pressure control and other measures, collectively termed "supportive care", constitute a mainstay of therapy [2,3] before immunosuppressive strategies may be considered [4].

Blood pressure increases very early in IgAN patients and even seemingly normotensive patients have higher blood pressures than matched healthy controls and exhibit subtle cardiac changes [5]. In addition, there is considerable evidence of early activation of the renin-angiotensin system (RAS) in the kidneys of IgAN patients [6, 7], and this has been related to the pathogenesis of tubulointerstitial damage [8]. Combined with the well-established antiproteinuric action of RAS blockers, this lays the basis for a strong recommendation to initiate and up-titrate ACE-inhibitors or angiotensin-receptor blockers (ARB) in all proteinuric IgAN patients at risk for progressive renal disease [2]. This 
recommendation is backed by randomized controlled clinical trials in IgAN showing better renal outcome in patients treated with enalapril versus non-RAS blocker antihypertensives [9] or benazepril versus placebo [10].

Already 18-20 years ago small clinical trials in IgAN patients reported that the combination of losartan with an ACE-inhibitor exerts additive antiproteinuric effects that were independent of achieved blood pressures $[11,12]$. This was then confirmed and extended to various other glomerular diseases [13-15]. However, subsequent major clinical trials focusing on renal and/or cardiovascular outcome in diabetic patients failed to detect a benefit from combined ARB - ACE inhibitor therapy in diabetic kidney disease but rather noted a higher risk of acute kidney injury and hyperkalemia with the combination as compared to the single substances $[16,17]$. The higher prevalence of such adverse events may relate to a too rapid up-titration to maximal doses with consecutive extra- and intrarenal vascular changes in diabetics that result in manifest or functional renal artery stenosis and thus a pronounced fall in renal perfusion with dual RAS blockade and subsequent acute renal injury. It is currently unknown whether this problem also occurs in primary glomerular diseases such as IgAN, in particular since it usually manifests in younger adults with relatively low atherosclerotic burden. In the present analysis we aimed to analyze whether renal endpoints including courses of renal function and proteinuria were affected by single or dual RAS blockade in patients that were included in the randomized controlled STOP-IgAN trial [3].

\section{METHODS}

\section{Main STOP-IgAN trial}

The STOP-IgAN trial was a multicenter, open label, randomized, controlled study that recruited 337 patients with biopsy-proven IgA nephropathy between February 2008 and October 2011. Protocol and results from the original trial have been published $[3,18]$. Briefly, eligible patients entered a 6-month run-in phase with comprehensive optimization of supportive treatment strategies including antihypertensive therapy with RAS blockers targeting a blood pressure below 125/75 mmHg. Additional measures comprised dietary counseling, cholesterol lowering, education to quit smoking, and avoidance of nonsteroidal anti-inflammatory drugs and other nephrotoxins. Upon completion of the run-in phase, 162 patients with persistent proteinuria $>0.75 \mathrm{~g} /$ day, but less than $3.5 \mathrm{~g} /$ day, despite optimized supportive care were then randomized into the following 3-year trial phase and were assigned to either continue on supportive therapy alone or to receive additional immunosuppression. Co-primary hierarchically ordered endpoints of the STOP-IgAN trial comprised achievement of full clinical remission (i.e. proteinuria below $0.2 \mathrm{~g} / \mathrm{g}$ and eGFR-loss less than $5 \mathrm{ml} /$ $\mathrm{min} / 1.73 \mathrm{~m}^{2}$ at the end of the 3-year trial phase) and eGFRloss $\geq 15 \mathrm{ml} / \mathrm{min} / 1.73 \mathrm{~m}^{2}$ over the trial phase. Rates of eGFRloss $\geq 30 \mathrm{ml} / \mathrm{min} / 1.73 \mathrm{~m}^{2}$ and onset of ESRD during the trial phase were captured as secondary endpoints.

\section{Study design and participants}

Among the entire cohort of 162 randomized STOP-IgAN participants, available endpoint information and a complete data set on RAS blocker treatment (i.e. at the time of randomization as well as 12, 24 and 36 months after randomization) were available for 112 patients. These individuals were classified post hoc based on their individual RAS blocker treatment regimen into patients on continuous single or dual RAS blocker therapy. A distinction between different ACE inhibitor or ARB substances was not made. Among the originally randomized cohort, 50 patients were not included in the present analysis due to missing information on RAS $(n=32)$ treatment at any of the indicated timepoints or since RAS treatment strategy had been interrupted $(n=7)$ or changed from single to dual RAS blockade $(n=2)$ or vice versa $(n=9)$. Systolic and diastolic blood pressure was recorded as office blood pressure measured at the time of randomization as well as 12, 24 and 36 months after randomization. Detailed information on antihypertensive treatment was captured at randomization and upon completion of the 3-year trial phase.

\section{Serum aldosterone assessment}

Serum aldosterone levels were measured in samples obtained at the end of the trial phase using the Parameter ${ }^{\circledR}$ Aldosterone Assay (R\&D Systems, Biotechne, Abingdon, UK) following the manufacturer's instructions in a threefold dilution.

\section{Statistical analyses}

All statistical analyses were performed with SAS 9.4 Software (SAS Institute Inc., Cary, NC, USA). Data are presented as mean \pm standard deviations for continuous variables and as counts and percentages for binary variables. To test the occurrence of the binary primary and secondary endpoints of the main STOP-IgAN trial in the two RAS blocker treatment groups, we applied a logistic regression model (proc LOGISTIC in SAS) adjusting for age, baseline GFR ( $\geq 60$ vs. $<60 \mathrm{ml} / \mathrm{min} / 1.73 \mathrm{~m}^{2}$ ) and proteinuria $(<1.5$ vs. $\geq 1.5 \mathrm{~g}$ /day) as well as the treatment arm (supportive care vs. supportive care plus immunosuppression) during the 3-year trial phase. Due to quasi complete separation in 
the analyses of the events "eGFR-loss $\geq 30 \mathrm{ml} / \mathrm{min} / 1.73 \mathrm{~m}^{2}$ " and "ESRD" the Firth correction was applied.

The course of proteinuria over the 3-year trial phase was analyzed using a linear mixed effects model with random intercept and slope (proc MIXED in SAS). As fixed effects we modeled age, baseline GFR ( $\geq 60 \mathrm{vs}$. $<60 \mathrm{ml} / \mathrm{min} / 1.73$ $\mathrm{m} 2)$ and proteinuria ( $<1.5 \mathrm{vs} . \geq 1.5 \mathrm{~g} /$ day $)$, treatment and RAS blocker treatment. We used the variance components covariance structure and adjusted the degrees of freedom by the "between-and-within" method. The residual plots were examined visually to assess the model fit and extreme outliers were excluded based on the restricted likelihood distance. Although this is an explorative evaluation, $p$ values $\leq 0.05$ were categorized as significant.

\section{RESULTS}

\section{Intervention groups and baseline characteristics}

A complete data set on renal function, proteinuria, study endpoints and RAS treatment over the entire 3.5 years was

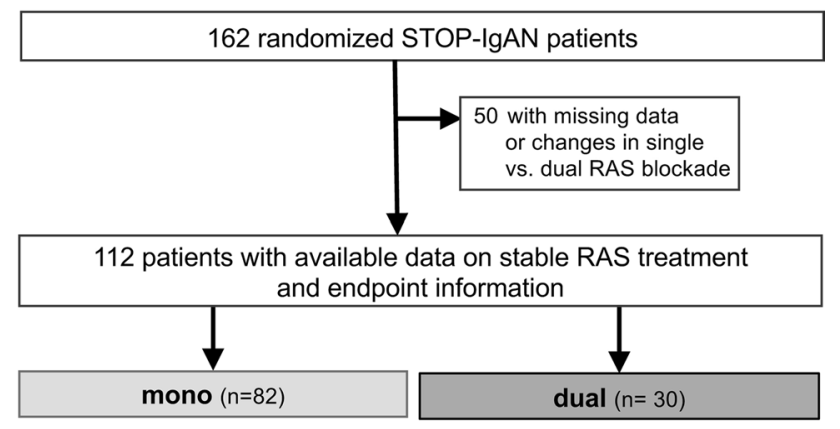

Fig. 1 Flowchart of analyzed patients available for 112 patients (i.e. $69 \%$ of the 162 patients who completed the original STOP-IgAN trial). Among these patients, 82 (73\%) stably received single RAS blocker therapy and $30(27 \%)$ patients received continuous dual RAS blocker therapy during the trial phase (Fig. 1). Patients' demographic and clinical characteristics at enrollment into the trial are given in Table 1. Treatment allocation to either continue supportive care or receive additional immunosuppression during the subsequent 3 -year trial phase was comparable between the two RAS blocker intervention groups. Patients on dual RAS blockade were equally distributed across the participating centers.

Table 1 also shows that the 50 patients who were excluded from the present analysis exhibited comparable baseline characteristics to those of the included patients.

\section{Renal outcomes in the different RAS intervention groups}

In both RAS blocker intervention groups, the occurrence of any of the primary and secondary renal endpoints at the end of the subsequent 3-year trial phase, i.e. achievement of full clinical remission, eGFR-loss of $\geq 15$ or $\geq$ $30 \mathrm{ml} / \mathrm{min} / 1.73 \mathrm{~m}^{2}$ and onset of ESRD did not differ significantly (Table 2). Patients on stable dual RAS blocker therapy moderately increased their proteinuria by $0.1 \mathrm{~g} / \mathrm{g}$, whereas patients on stable single RAS blocker therapy significantly decreased their proteinuria by $0.3 \mathrm{~g} / \mathrm{g}$ over the study period (Fig. 2a). Multivariate analyses demonstrated that both the RAS strategy $(p=0.011)$ as well as treatment allocation to either supportive care alone or additional immunosuppression $(\mathrm{p}=0.039)$ significantly affected the course of proteinuria over the trial phase (Table 3). A further sensitivity analysis that also included patients for whom RAS blocker information was available
Table 1 Baseline characteristics of trial participants at randomization based on stratification of RAS blockade regimen during the STOP-IgAN trial

\begin{tabular}{llll}
\hline Subgroup & $\begin{array}{l}\text { Single } \\
\text { RAS blockade }\end{array}$ & $\begin{array}{l}\text { Dual } \\
\text { RAS blockade } \\
(\mathrm{n}=82)\end{array}$ & $\begin{array}{l}\text { Excluded } \\
\text { Patients } \\
(\mathrm{n}=30)\end{array}$ \\
\hline Female sex (\%) & 28 & 13 & 16 \\
Smoker (\%) & 18 & 21 & 13 \\
Age (years) & $45.5 \pm 12.2$ & $44.5 \pm 12.8$ & $43.8 \pm 13.1$ \\
Body mass index $\left(\mathrm{kg} / \mathrm{m}^{2}\right)$ & $27.8 \pm 5.3$ & $29.8 \pm 6.2$ & $26.8 \pm 4.1$ \\
Blood pressure $(\mathrm{mmHg})$ & $126 \pm 14$ & $134 \pm 18$ & $132 \pm 15$ \\
systolic & $77 \pm 11$ & $81 \pm 11$ & $83 \pm 12$ \\
diastolic & & & \\
GFR (ml/min) & $76.8 \pm 32.6$ & $79.1 \pm 33.6$ & $74.4 \pm 36.5$ \\
Urinary protein excretion $(\mathrm{g} / \mathrm{d})$ & $1.6 \pm 0.8$ & $1.7 \pm 0.8$ & $1.8 \pm 0.7$ \\
Urinary protein-to-creatinine ratio $(\mathrm{g} / \mathrm{g})$ & $1.0 \pm 0.9$ & $1.0 \pm 0.6$ & $1.1 \pm 0.6$ \\
Patients randomized to supportive care in the & 52 & 53 & 42 \\
$\quad$ trial phase $(\%)$ & & & \\
\hline
\end{tabular}


Table 2 Primary and secondary trial endpoints of analyzed patients in the RAS treatment groups

\begin{tabular}{|c|c|c|c|c|c|}
\hline \multirow[t]{2}{*}{ Subgroup } & \multicolumn{2}{|c|}{ Single RAS blockade } & \multicolumn{2}{|c|}{ Dual RAS blockade } & \multirow[t]{2}{*}{$p$ value (adjusted) } \\
\hline & $\mathrm{N}$ & $\begin{array}{l}\text { mean } \pm \mathrm{SD} \\
\text { or no. }(\%)\end{array}$ & $\mathrm{N}$ & $\begin{array}{l}\text { mean } \pm \text { SD } \\
\text { or no. }(\%)\end{array}$ & \\
\hline In full clinical remission & 78 & $12(15)$ & 30 & $4(13)$ & 0.920 \\
\hline eGFR-drop $\geq 15 \mathrm{ml} / \mathrm{min} / 1.73 \mathrm{~m}^{2}$ & 82 & $15(18)$ & 30 & $5(17)$ & 0.692 \\
\hline eGFR-drop $\geq 30 \mathrm{ml} / \mathrm{min} / 1.73 \mathrm{~m}^{2}$ & 82 & $2(2)$ & 30 & $2(7)$ & 0.405 \\
\hline ESRD onset & 82 & $1(6)$ & 30 & $0(0)$ & 0.813 \\
\hline
\end{tabular}

at only two out of three time points (i.e. 12, 24 and 36 months upon randomization) contributed 20 additional patients to the analysis (with only 30 remaining STOPIgAN patients without such information) and entirely confirmed the primary analysis (data not shown). Estimated GFR and proteinuria courses separated by RAS therapy and treatment allocation to either supportive care alone or additional immunosuppression are given in Table 4. A decrease in proteinuria by $0.5 \mathrm{~g} / \mathrm{g}$ over the trial phase was observed in patients under single RAS inhibition and additional immunosuppression. There was an increase in proteinuria in patients receiving dual RAS blockade, but no additional immunosuppression. Estimated GFRs remained almost stable over the entire STOP-IgAN trial in both RAS intervention groups between enrollment and end of the trial (Fig. 2b).

\section{Blood pressure changes and antihypertensive management during the trial phase}

At the time of randomization, mean systolic blood pressure was higher in patients under dual RAS blockade as compared to those under single RAS therapy (Table 5). Yet, mean diastolic blood pressure levels did not differ. Over the subsequent 3-year trial phase, blood pressure levels remained stable in both arms without obvious differences between the two RAS intervention groups. At randomization, as well as at the end of the trial phase, a higher number of patients under dual RAS blockade more commonly received more than 3 antihypertensive agents than those under single RAS blockade. However, there were no obvious differences in the numbers of antihypertensive agents being increased, decreased or kept on a stable level between the two RAS intervention arms (Table 5). The number of patients under maximum allowed ACE inhibitor therapy according to prescribing information remained unchanged under dual RAS blockade over the study period. By contrast, ARB dosage had been maximized at the end of the 3-year trial phase in four additional patients on single RAS blockade compared to two patients on a dual RAS blocker therapy strategy (Table 5).

\section{Serum aldosterone levels during the trial phase}

To assess potential mechanisms accounting for the increase in proteinuria with dual RAS blockade, we measured aldosterone levels in patients with available serum samples obtained at the end of the trial (in 57 patients under single and 20 patients under dual RAS blockade). Aldosterone levels were suppressed, i.e. below the detection limit (i.e. $22 \mathrm{pg} /$ $\mathrm{ml}$ ), in $56 \%$ of the analyzed samples and detectable values did not differ between patients under continuous single or dual RAS blockade over the trial phase (Fig. 3).

\section{DISCUSSION}

There is no doubt that inhibition of the RAS system is the most widely accepted treatment to achieve blood pressure and proteinuria targets in IgAN patients and that it is one of the major pillars of supportive treatment in these patients [2, 9, 19-21]. Years ago, smaller studies, mostly in patients with diabetes, suggested favorable anti-proteinuric effects from an ACE inhibitor/ARB combination [22] and dual RAS blockade was also applied to other patients. Yet, further evidence from clinical trials and meta-analyses demonstrated that dual RAS blockade provoked serious side effects including severe hypotension, hyperkalemia and renal failure in diabetic patients [17, 23, 24]. The randomized ONTARGET study did not show improved renal and cardiovascular outcomes under a fixed and rapid up-titration of dual RAS blockade over a run-in period of 3-4 weeks in patients at risk for cardiovascular disease. ONTARGET participants under dual RAS blockade even exhibited an increased rate of acute renal failure in this cohort [16]. Other randomized trials, such as LIRICO, VALID and PREPARE-2 comparing single with dual RAS blockade did not show deterioration of renal function in the dual approach but failed to detect renal benefits in diabetic and non-diabetic patients [25-27]. Current KDIGO guidelines do not recommend the use of dual RAS blockade in proteinuric IgAN patients since there is insufficient evidence to prove renal benefits from such strategy [2]. In 2014, the EMA's Committee for Medicinal Products for Human Use even endorsed restrictions for the use of a dual RAS blockade [28]. 


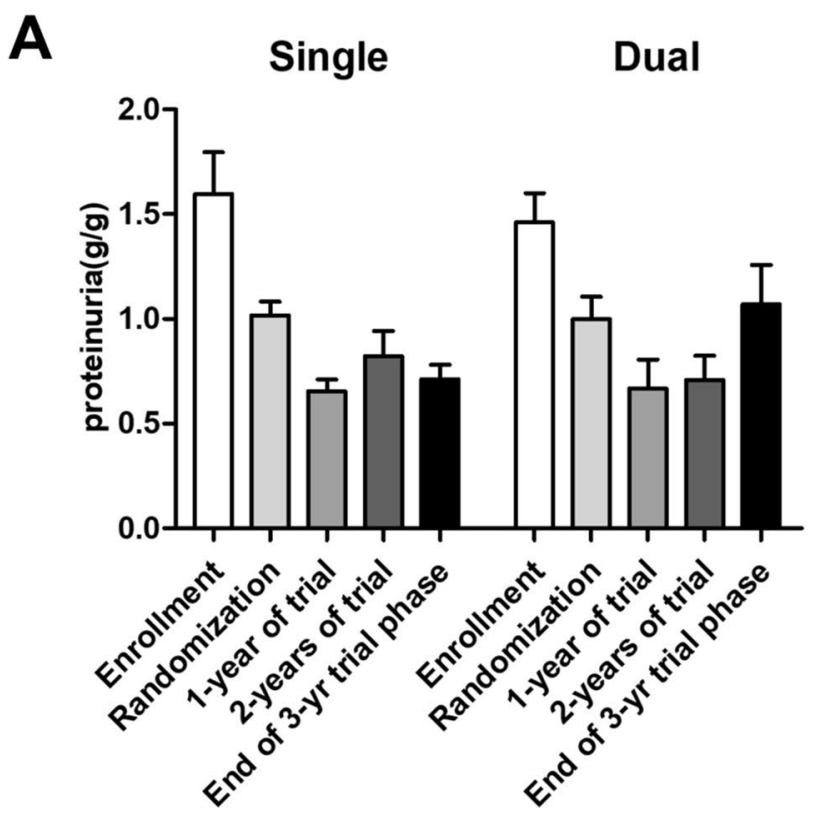

B

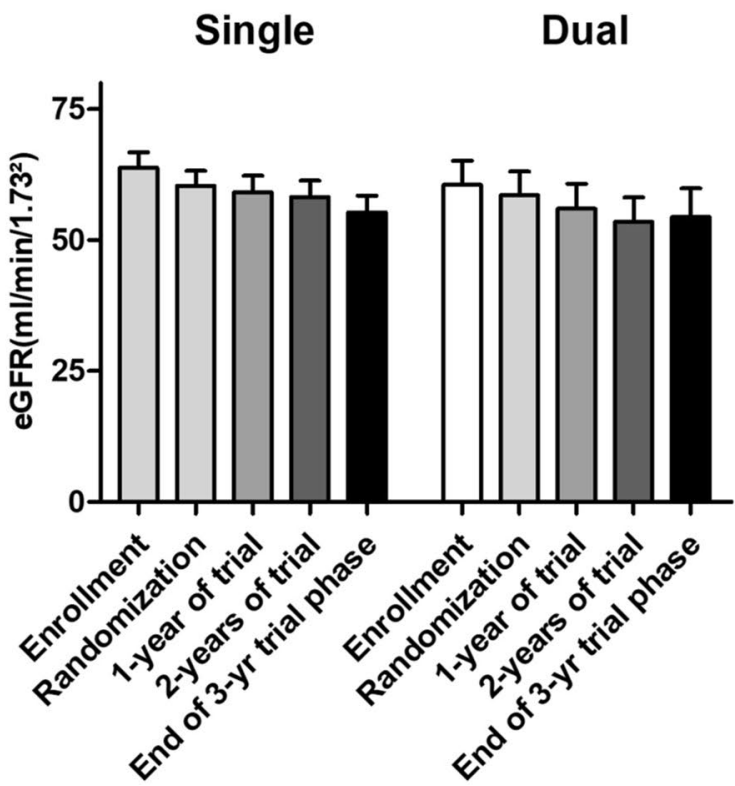

Fig. 2 Urinary protein excretion (a) and eGFR (b) at enrollment, at randomization (i.e. at the end of the 6-month run-in phase) and at the end of the 3-year trial phase in patients under single versus dual RAS blockade

Despite the above, the use of dual RAS blockade in IgAN and other glomerulonephritides is a matter of ongoing debate. In particular, it has been argued that patients with IgAN and many other types of glomerulonephritis are often younger adults and do not exhibit widespread cardiovascular disease, in particular stenosing atherosclerotic vessel damage, which in turn might predispose patients to acute kidney injury in the case of dual RAS blockade.

The STOP IgAN trial was conducted from 2008 to 2011 at 32 German centers. About $27 \%$ of all patients from the trial phase received dual RAS blocker therapy throughout the trial. In the present post hoc analysis, we investigated the association of dual versus single RAS blocker strategy on renal outcome parameters. None of the primary and secondary STOP-IgAN outcome measures, such as full clinical remission, eGFR-drop rates and ESRD occurrence, were affected by the RAS blocker regimen. Patients under dual RAS blockade even exhibited an increase in mean proteinuria to the end of the trial phase as compared to patients under single RAS blockade, in whom proteinuria decreased. Mean eGFR in both groups remained stable over the entire trial.

Reasons to initiate dual RAS blockade in IgAN patients at risk for a progressive disease course might be attempts to lower blood pressure as well as proteinuria and thus renal risk. However, at least with regard to proteinuria, our data rather disprove a long-lasting anti-proteinuric effect of dual RAS blockade. Multivariate analyses demonstrated that the RAS treatment strategy during our trial phase (i.e. single vs. dual inhibition) as well as treatment allocation to supportive care alone or additional immunosuppression, significantly affected the course of proteinuria. Patients under single RAS blockade and additional immunosuppression exhibited decreased proteinuria over the trial phase, whereas those under dual RAS blockade but with no additional immunosuppression had increased proteinuria. While the former could point to an improved long-term outcome, our very recent analysis of renal outcomes of the STOP-IgAN cohort after a mean follow-up of 7.4 years did not indicate any lasting benefit of immunosuppression on an endpoint composed of death, dialysis and 40\% eGFR loss [29].

The observed increase in proteinuria in patients under dual RAS blockade at the end of the 3-year trial phase was unexpected and cannot be concisely explained based on the available data and our analysis strategy. Of note, systolic blood pressure at randomization tended to be higher in the "dual RAS blockade" group, however, during the randomized trial phase the courses of blood pressure levels were comparable between the two arms. In addition, we did not find elevated serum aldosterone levels in patients under dual RAS blockade as compared to those under single therapy. Increased serum aldosterone has been described for proteinuric patients with diabetic nephropathy under single ARB therapy [30]. Currently, mineralocorticoid receptor antagonists are primarily recommended as a second-line alternative in patients who do not tolerate ACEi or ARBs. Alternatively, we cannot exclude a possible selection bias. Despite attempts to account for this statistically, we cannot completely exclude that the increased proteinuria in patients under dual RAS blockade was due to slightly higher average 
Table 3 Annual change in proteinuria since randomization (linear mixed model)

\begin{tabular}{lrcc}
\hline Annual change in proteinuria $(\mathrm{g} / \mathrm{g})$ & & \\
\hline Predictor & Estimate & $95 \% \mathrm{CI}$ & $p$ value \\
\hline RAS treatment strategy & -0.163 & $-0.287-0.039$ & 0.011 \\
Age & 0.001 & $-0.004-0.005$ & 0.765 \\
Time & -0.024 & $-0.065-0.017$ & 0.254 \\
Baseline GFR $\geq$ or $\left(<60 \mathrm{ml} / \mathrm{min} / 1.73 \mathrm{~m}^{2}\right)$ & -0.267 & $-0.388-0.146$ & $<0.0001$ \\
Baseline proteinuria $(<1.5$ or $\geq 1.5 \mathrm{~g} / \mathrm{day})$ & -0.406 & $-0.518-0.294$ & $<0.0001$ \\
Treatment during trial phase $($ SUP vs. IMM) & 0.115 & $0.006-0.224$ & 0.039 \\
\hline
\end{tabular}

Table 4 Course of renal function and proteinuria, depending on continuous RAS treatment strategy over the randomized 3-year trial phase

\begin{tabular}{|c|c|c|c|c|c|c|c|}
\hline & & \multicolumn{3}{|c|}{ eGFR $\left(\mathrm{ml} / \mathrm{min} / 1.73 \mathrm{~m}^{2}\right)$} & \multicolumn{3}{|c|}{ Urinary protein-to-creatinine ratio $(\mathrm{g} / \mathrm{g})$} \\
\hline & & $N$ & At randomization & End of trial phase & $N$ & At randomization & End of trial phase \\
\hline \multirow{2}{*}{$\begin{array}{l}\text { Single RAS blockade dur- } \\
\text { ing trial phase }\end{array}$} & Supportive care & 43 & $58.2 \pm 25.7$ & $51.1 \pm 29.5$ & 41 & $1.0 \pm 0.5$ & $0.8 \pm 0.6$ \\
\hline & $\begin{array}{l}\text { Additional immunosup- } \\
\text { pression }\end{array}$ & 39 & $62.7 \pm 27.1$ & $59.9 \pm 28.0$ & 37 & $1.1 \pm 0.6$ & $0.6 \pm 0.6$ \\
\hline \multirow{2}{*}{$\begin{array}{l}\text { Dual RAS blockade during } \\
\text { trial phase }\end{array}$} & Supportive care & 16 & $59.7 \pm 29.8$ & $55.3 \pm 38.0$ & 16 & $0.9 \pm 0.5$ & $1.1 \pm 0.8$ \\
\hline & $\begin{array}{l}\text { Additional immunosup- } \\
\text { pression }\end{array}$ & 14 & $57.4 \pm 18.3$ & $53.4 \pm 18.6$ & 14 & $1.2 \pm 0.7$ & $1.0 \pm 1.2$ \\
\hline
\end{tabular}

blood pressures (Table 5) or higher IgAN disease activity. However, at least the baseline proteinuria levels were similar in both groups suggesting that there was no systematic selection bias.

Our study is limited by its post hoc character and the small sample size. Of note, the original trial was not powered to detect differences in proteinuria between patients under single and dual RAS blocker treatment. Third, in the present analysis we experienced a significant loss of 50 originally randomized patients who were not included in the present post hoc analysis due to missing data or, more importantly, due to changes in RAS therapy strategy. However, the excluded patients from the present analysis had comparable baseline characteristics versus those who had been included. Lastly, we cannot exclude a selection bias since decisions on single or dual RAS treatment regimen were based upon the physician's discretion in the clinical routine and did not follow a protocol-defined algorithm. At the time of enrollment, patients under dual RAS blockade tended to have higher mean systolic blood pressure, received more antihypertensives and tended to have an increased body mass index than those under single RAS inhibition suggesting that these patients per se exhibited features of an unfavorable natural course of their IgAN. However, in the present secondary analysis, none of these differences achieved statistical significance.

Our results from this secondary analysis of a STOPIgAN subcohort shed new light on the role of dual RAS inhibition in patients with $\operatorname{IgAN}$, yet we cannot exclude some degree of selection bias. Consistent with numerous prior RCTs, predominantly in diabetic nephropathy, we failed to obtain evidence that dual RAS blockade exerted beneficial renal effects in IgAN. Rather, patients on this regimen even exhibited higher proteinuria at the end of the randomized 3-year trial phase, independent of the STOP IgAN treatment allocation to supportive care alone or additional immunosuppression. Our data thereby support the current approach backed by KDIGO guidelines (www.kdigo.org) to up-titrate individual RAS blockers to maximally allowed or tolerated dosages rather than combining them. 
Table 5 Course of blood pressure (bp) and antihypertensive treatment in the RAS treatment groups

\begin{tabular}{|c|c|c|c|c|}
\hline \multirow[t]{2}{*}{ Subgroup } & \multicolumn{2}{|c|}{ Single RAS blockade $(n=112)$} & \multicolumn{2}{|c|}{ Dual RAS blockade $(n=30)$} \\
\hline & $N$ & Mean \pm SD or no. $(\%)$ & $N$ & Mean \pm SD or no. $(\%)$ \\
\hline \multicolumn{5}{|l|}{ Blood pressure $(\mathrm{mmHg})$} \\
\hline \multicolumn{5}{|l|}{ At randomization } \\
\hline Systolic & 80 & $125.5 \pm 13.7$ & 29 & $134.1 \pm 18.5$ \\
\hline Diastolic & & $77.5 \pm 10.8$ & & $80.7 \pm 10.7$ \\
\hline \multicolumn{5}{|l|}{12 months after randomization } \\
\hline Systolic & 81 & $127.1 \pm 14.2$ & 30 & $128.8 \pm 10.7$ \\
\hline Diastolic & & $78.8 \pm 9.1$ & & $79 \pm 8.6$ \\
\hline $\begin{array}{l}24 \text { months after randomization } \\
\text { Systolic } \\
\text { Diastolic }\end{array}$ & 82 & $\begin{array}{l}129.1 \pm 15.6 \\
81.3 \pm 9.6\end{array}$ & 29 & $\begin{array}{l}133.4 \pm 17.5 \\
79.5 \pm 10.1\end{array}$ \\
\hline $\begin{array}{l}36 \text { months after randomization } \\
\text { Systolic } \\
\text { Diastolic }\end{array}$ & 81 & $\begin{array}{l}127.7 \pm 16.2 \\
80.7 \pm 12.6\end{array}$ & 30 & $\begin{array}{l}131.1 \pm 17.9 \\
81.8 \pm 10.6\end{array}$ \\
\hline \multicolumn{5}{|c|}{ Antihypertensive agents No. (\% of subgroup) } \\
\hline $\begin{array}{l}\text { At randomization } \\
1 \mathrm{bp} \text { medication } \\
2 \mathrm{bp} \text { medications } \\
3 \mathrm{bp} \text { medications } \\
>3 \text { bp medications }\end{array}$ & 82 & $\begin{array}{l}21(26) \\
23(28) \\
13(15.9) \\
25(30.5)\end{array}$ & 30 & $\begin{array}{l}0(0) \\
10(23.3) \\
4(16.7) \\
16(53.3)\end{array}$ \\
\hline $\begin{array}{l}36 \text { months after randomization } \\
1 \text { bp medication } \\
2 \text { bp medications } \\
3 \text { bp medications } \\
>3 \text { bp medications }\end{array}$ & 82 & $\begin{array}{l}15(18.3) \\
21(25.6) \\
12(14.6) \\
34(41.5)\end{array}$ & 30 & $\begin{array}{l}0(0) \\
7(23.3) \\
5(16.7) \\
18(60.0)\end{array}$ \\
\hline \multicolumn{5}{|c|}{ Adjustment of antihypertensive drugs over the 3-year trial } \\
\hline Increased & 82 & $27(32.9)$ & 30 & $11(36.7)$ \\
\hline Unchanged & 82 & $44(53.7)$ & 30 & $16(53.3)$ \\
\hline Decreased & 82 & $11(13.4)$ & 30 & $3(10)$ \\
\hline \multicolumn{5}{|l|}{ Maximum ACE dosage } \\
\hline At randomization & 82 & $30(36.6)$ & 30 & $17(56.7)$ \\
\hline 36 months after randomization & 82 & $23(28.1)$ & 30 & $17(56.7)$ \\
\hline \multicolumn{5}{|l|}{ Maximum ARB dosage } \\
\hline At randomization & 82 & $20(24.4)$ & 30 & $12(40)$ \\
\hline 36 months after randomization & 82 & $24(29.3)$ & 30 & $14(46.7)$ \\
\hline
\end{tabular}


Fig. 3 Aldosterone measurements in available serum samples obtained at the end of the trial phase according to continuous single or dual RAS blocker therapy during the randomized 3-year trial phase

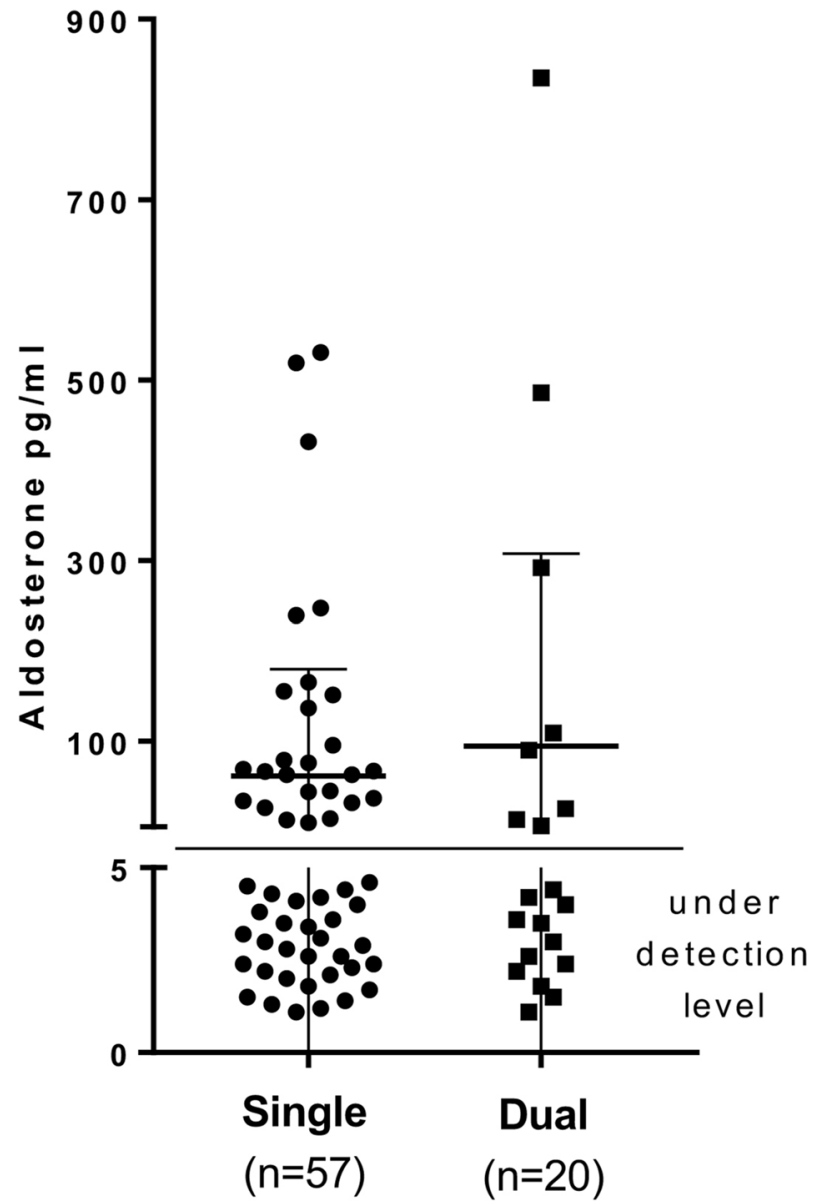

Single RAS blockade

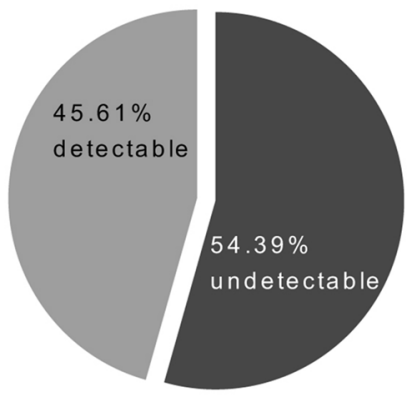

$\mathrm{n}=57$
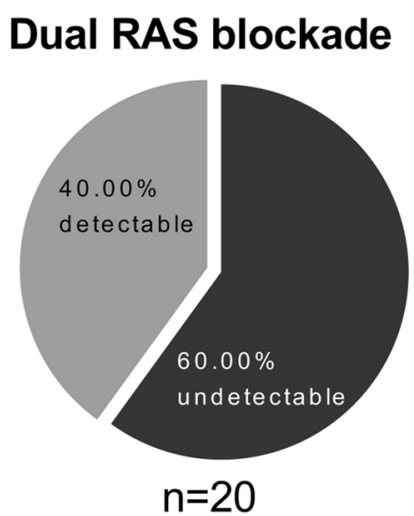

Acknowledgements We thank all the patients and trial centers that participated in the STOP-IgAN trial (www.ClinicalTrials.gov, NCT00554502).

Author contributions The study was designed by J.F., F.E., R.D.H. and T.R. Statistical analyses were performed by D.P.L., C.S., S.W. and C.F. and R.D.H. D.P.L. wrote the entire manuscript. C.S., J.F., R.D.H. and T.R. edited the manuscript text. The final manuscript version was approved by all listed authors.

Funding The original STOP-IgAN trial was funded by the German Federal Ministry of Education and Research funded the trial (GFVT01044604). There was no extra funding for this secondary analysis. Open Access funding provided by Projekt DEAL.

\section{Compliance with ethical standards}

Conflict of interest J.F. has received consultant honoraria from Omeros, USA, Retrophin, USA and Calliditas, Sweden. The other authors declare no competing financial interests.

Ethics approval The STOP-IgAN trial was performed in line with with the principles of the 1964 Declaration of Helsinki and approval was granted by the Ethics Committees at each participating center (www. ClinicalTrials.gov, NCT00554502).
Research involving Human Participants and/or Animals The STOPIgAN trial was conducted as a multicenter, open label, randomized, controlled study between February 2008 and October 2011 (www. ClinicalTrials.gov, NCT00554502). All data presented in this secondary analysis were recorded as detailed in the study protocol [3]. The STOP-IgAN trial was performed in line with the principles of the 1964 Declaration of Helsinki and approval was granted by the Ethics Committees at each participating center.

Informed consent Written informed consent was obtained from all individual participants included in the STOP-IgAN trial prior to trial enrollment. This consent included collection of serum samples.

Open Access This article is licensed under a Creative Commons Attribution 4.0 International License, which permits use, sharing, adaptation, distribution and reproduction in any medium or format, as long as you give appropriate credit to the original author(s) and the source, provide a link to the Creative Commons licence, and indicate if changes were made. The images or other third party material in this article are included in the article's Creative Commons licence, unless indicated otherwise in a credit line to the material. If material is not included in the article's Creative Commons licence and your intended use is not permitted by statutory regulation or exceeds the permitted use, you will need to obtain permission directly from the copyright holder. To view a copy of this licence, visit http://creativecommons.org/licenses/by/4.0/. 


\section{References}

1. Wyatt RJ, Julian BA (2013) IgA nephropathy. N Engl J Med 368(25):2402-2414

2. KDIGO Clinical Practice Guideline for Glomerulonephritis (2012) Kidney International Supplements 2(2):142

3. Rauen $\mathrm{T}$ et al (2015) Intensive Supportive Care plus Immunosuppression in IgA Nephropathy. N Engl J Med 373(23):2225-2236

4. Ma, F., et al., Treatment for IgA nephropathy with stage 3 or 4 chronic kidney disease: low-dose corticosteroids combined with oral cyclophosphamide. J Nephrol, 2020.

5. Stefanski A et al (1996) Early increase in blood pressure and diastolic left ventricular malfunction in patients with glomerulonephritis. Kidney Int 50(4):1321-1326

6. Nishiyama A et al (2011) Urinary angiotensinogen reflects the activity of intrarenal renin-angiotensin system in patients with IgA nephropathy. Nephrol Dial Transplant 26(1):170-177

7. Del Prete D et al (2003) Precocious activation of genes of the renin-angiotensin system and the fibrogenic cascade in IgA glomerulonephritis. Kidney Int 64(1):149-159

8. Chan LY et al (2005) Tubular expression of angiotensin II receptors and their regulation in IgA nephropathy. J Am Soc Nephrol 16(8):2306-2317

9. Praga M et al (2003) Treatment of IgA nephropathy with ACE inhibitors: a randomized and controlled trial. J Am Soc Nephrol 14(6):1578-1583

10. Coppo R et al (2007) IgACE: a placebo-controlled, randomized trial of angiotensin-converting enzyme inhibitors in children and young people with IgA nephropathy and moderate proteinuria. $\mathrm{J}$ Am Soc Nephrol 18(6):1880-1888

11. Russo D et al (1999) Additive antiproteinuric effect of converting enzyme inhibitor and losartan in normotensive patients with $\operatorname{IgA}$ nephropathy. Am J Kidney Dis 33(5):851-856

12. Russo D et al (2001) Coadministration of losartan and enalapril exerts additive antiproteinuric effect in IgA nephropathy. Am J Kidney Dis 38(1):18-25

13. Luno J et al (2002) Effects of dual blockade of the renin-angiotensin system in primary proteinuric nephropathies. Kidney Int Suppl 82:S47-52

14. Rossing $\mathrm{K}$ et al (2002) Dual blockade of the renin-angiotensin system in diabetic nephropathy: a randomized double-blind crossover study. Diabetes Care 25(1):95-100

15. Jacobsen $P$ et al (2003) Additive effect of ACE inhibition and angiotensin II receptor blockade in type I diabetic patients with diabetic nephropathy. J Am Soc Nephrol 14(4):992-999

16. Mann JF et al (2008) Renal outcomes with telmisartan, ramipril, or both, in people at high vascular risk (the ONTARGET study): a multicentre, randomised, double-blind, controlled trial. Lancet 372(9638):547-553

17. Fried LF et al (2013) Combined angiotensin inhibition for the treatment of diabetic nephropathy. N Engl J Med 369(20):1892-1903
18. Eitner F et al (2008) Supportive Versus Immunosuppressive Therapy of Progressive IgA nephropathy (STOP) IgAN trial: rationale and study protocol. J Nephrol 21(3):284-289

19. Cattran DC, Greenwood C, Ritchie S (1994) Long-term benefits of angiotensin-converting enzyme inhibitor therapy in patients with severe immunoglobulin a nephropathy: a comparison to patients receiving treatment with other antihypertensive agents and to patients receiving no therapy. Am J Kidney Dis 23(2):247-254

20. Li PK et al (2006) Hong Kong study using valsartan in IgA nephropathy (HKVIN): a double-blind, randomized, placebocontrolled study. Am J Kidney Dis 47(5):751-760

21. Coppo, R. and T. Robert, IgA nephropathy in children and in adults: two separate entities or the same disease? J Nephrol, 2020.

22. Kunz R et al (2008) Meta-analysis: effect of monotherapy and combination therapy with inhibitors of the renin angiotensin system on proteinuria in renal disease. Ann Intern Med 148(1):30-48

23. Makani H et al (2013) Efficacy and safety of dual blockade of the renin-angiotensin system: meta-analysis of randomised trials. BMJ 346:f360

24. Palmer SC et al (2015) Comparative efficacy and safety of blood pressure-lowering agents in adults with diabetes and kidney disease: a network meta-analysis. Lancet 385(9982):2047-2056

25. Saglimbene V et al (2018) The Long-Term Impact of ReninAngiotensin System (RAS) Inhibition on Cardiorenal Outcomes (LIRICO): A Randomized. Controlled Trial J Am Soc Nephrol 29(12):2890-2899

26. Ruggenenti P et al (2019) Effects of valsartan, benazepril and their combination in overt nephropathy of type 2 diabetes: A prospective, randomized, controlled trial. Diabetes Obes Metab 21(5):1177-1190

27. Voskamp PWM et al (2017) Effect of dual compared to no or single renin-angiotensin system blockade on risk of renal replacement therapy or death in predialysis patients: PREPARE-2 study. J Am Soc Hypertens 11(10):635-643

28. Combined use of medicines affecting the renin-angiotensin system (RAS) to be restricted-CHMP endorses PRAC recommendation, in https://www.ema.europa.eu/en/glossary/chmp. 2014.

29. Rauen, T., et al., After ten years of follow-up, no difference between supportive care plus immunosuppression and supportive care alone in IgA nephropathy. Kidney Int, 2020.

30. Moranne $\mathrm{O}$ et al (2013) Determinants and changes associated with aldosterone breakthrough after angiotensin II receptor blockade in patients with type 2 diabetes with overt nephropathy. Clin J Am Soc Nephrol 8(10):1694-1701

Publisher's Note Springer Nature remains neutral with regard to jurisdictional claims in published maps and institutional affiliations. 